\title{
Long-term graft patency after replacement of the brachiocephalic veins combined with resection of mediastinal tumors
}

\author{
Yasushi Shintani, MD \\ Mitsunori Ohta, MD \\ Masato Minami, MD \\ Hiroyuki Shiono, MD \\ Hirohisa Hirabayashi, MD \\ Masayoshi Inoue, MD \\ Goro Matsumiya, MD \\ Hikaru Matsuda, MD
}

From the Department of General Thoracic Surgery, E1, Osaka University Graduate School of Medicine, Osaka, Japan.

Received for publication Feb 25, 2004; revisions requested April 20, 2004; accepted for publication May 4, 2004.

Address for reprints: Mitsunori Ohta, MD, Department of General Thoracic Surgery, E1, Osaka University Graduate School of Medicine, 2-2 Yamadaoka, Suita, Osaka 565-0871, Japan (E-mail: ohta@ surg1.med. osaka-u.ac.jp)

J Thorac Cardiovasc Surg 2005;129:809-12 $0022-5223 / \$ 30.00$

Copyright (C) 2005 by The American Association for Thoracic Surgery

doi:10.1016/j.jtcvs.2004.05.001
Objective: We sought to investigate the correlation between type of vascular reconstruction and long-term graft patency after replacement of brachiocephalic veins combined with resection of mediastinal malignancies.

Methods: Eighteen patients underwent surgical resection of tumors and the superior vena cava with concomitant vascular reconstruction using ringed polytetrafluoroethylene grafts. Graft patency was verified by means of venography or contrastenhanced computed tomography at time points ranging from 3 to 77 months (median, 33 months) postoperatively.

Results: Seven patients underwent sole reconstruction of the right brachiocephalic vein, with occlusion observed in only 1 patient. In 6 patients who underwent reconstruction of the bilateral brachiocephalic veins with 2 separate grafts, the grafts remained patent in 2, whereas 4 patients experienced occlusion of one of the two grafts yet remained asymptomatic. Both patients who underwent reconstruction with a Y graft experienced left brachiocephalic vein graft occlusion. In the 3 patients who underwent reconstruction of a left brachiocephalic vein, the graft became occluded, and superior vena cava syndrome developed in 2 of these patients.

Conclusion: When replacing the superior vena cava, reconstruction of a left brachiocephalic vein alone results in a significant rate of occlusion and development of superior vena cava syndrome. Thus we advocate sole right brachiocephalic vein reconstruction or bilateral brachiocephalic vein reconstruction in this setting, and separate reconstruction of the veins is preferable to use of a $\mathrm{Y}$ graft.

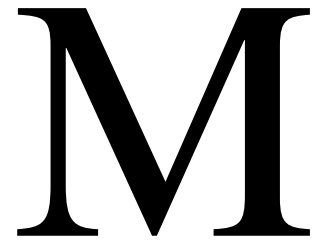

ediastinal tumors tend to invade the large vessels, nerves, lungs, and pericardium, and complete resection often requires reconstruction of the large vessels. Several investigators have reported favorable results when combining resection of malignant tissue with vascular reconstruction. ${ }^{1,2}$ For example, Dartevelle and colleagues ${ }^{3}$ reported good survivals after replacement of the superior vena cava (SVC) with a ringed polytetrafluoroethylene (PTFE) graft combined with resection of mediastinal-pulmonary malignant tumors. Ringed PTFE grafts have been reported to remain patent for several years ${ }^{4}$; however, the correlation between long-term graft patency and type of brachiocephalic vein $(\mathrm{BCV})$ reconstruction remains unknown. In the present study we characterized long-term graft patency on the basis of the $\mathrm{BCV}$ replacement procedure used. 
TABLE I. Clinicopathologic features of patients and reconstruction of BCVs

\begin{tabular}{|c|c|c|c|c|c|c|c|c|}
\hline Case no. & Age/sex & Diagnosis & $\begin{array}{l}\text { Reconstructed } \\
\text { vessels }\end{array}$ & PTFE graft & $\begin{array}{l}\text { Diameter } \\
\text { (mm) }\end{array}$ & $\begin{array}{c}\text { Graft } \\
\text { patency } \\
\text { (mo) }\end{array}$ & & $\begin{array}{l}\text { Occlusive } \\
\text { symptom }\end{array}$ \\
\hline 1 & $65 / F$ & Thymoma & Rt BCV & Single & 10 & Patent & 36 & - \\
\hline 2 & $53 / \mathrm{M}$ & Thymoma & Rt BCV & Single & 16 & Patent & 20 & - \\
\hline 3 & $26 / \mathrm{M}$ & Choriocarcinoma & Rt BCV & Single & 10 & Patent & 3 & - \\
\hline 4 & 19/M & Seminoma & Rt BCV & Single & 12 & Patent & 60 & - \\
\hline $5^{*}$ & $18 / \mathrm{M}$ & Embryonal carcinoma & Rt BCV & Single & 12 & Occlusive & 12 & - \\
\hline 6 & $42 / \mathrm{F}$ & Thymoma & Rt BCV & Single & 16 & Patent & 39 & + \\
\hline 7 & $57 / F$ & Thymoma & Rt BCV & Single & 10 & Patent & 77 & + \\
\hline 8 & $65 / F$ & Malignant teratoma & Lt BCV & Single & 12 & Patent & 8 & + \\
\hline 9 & $38 / F$ & Malignant Iymphoma & Lt BCV & Single & 12 & Occlusive & 11 & + \\
\hline $10^{*}$ & $53 / \mathrm{M}$ & Thymic cancer & Lt BCV & Single & 10 & Occlusive & 8 & - \\
\hline \multirow[t]{2}{*}{$11^{*}$} & $44 / F$ & Thymoma & Rt BCV & Separate & 10 & Patent & 44 & - \\
\hline & & & Lt BCV & & 8 & Patent & 44 & \\
\hline \multirow[t]{2}{*}{$12^{*}$} & $50 / \mathrm{M}$ & Thymoma & Rt BCV & Separate & 10 & Occlusive & 21 & - \\
\hline & & & Lt BCV & & 10 & Patent & 33 & \\
\hline \multirow[t]{2}{*}{13} & 77/M & Thymoma & Rt BCV & Separate & 10 & Patent & 26 & - \\
\hline & & & Lt BCV & & 10 & Occlusive & 26 & \\
\hline \multirow[t]{2}{*}{$14^{*}$} & $59 / \mathrm{M}$ & Thymic carcinoma & Rt BCV & Separate & 10 & Patent & 38 & + \\
\hline & & & Lt BCV & & 10 & Occlusive & 5 & \\
\hline \multirow[t]{2}{*}{$15^{*}$} & $67 / F$ & Thymic carcinoma & Rt BCV & Separate & 10 & Patent & 13 & + \\
\hline & & & Lt BCV & & 10 & Patent & 13 & \\
\hline \multirow[t]{2}{*}{$16^{*}$} & $32 / \mathrm{M}$ & Embryonal carcinoma & Rt BCV & Separate & 10 & Patent & 36 & - \\
\hline & & & Lt BCV & & 10 & Occlusive & 8 & \\
\hline \multirow[t]{2}{*}{17} & 74/M & Thymoma & Rt BCV & Y graft & 10 & Occlusive & 12 & + \\
\hline & & & Lt BCV & & 10 & Occlusive & 5 & \\
\hline \multirow[t]{2}{*}{18} & $47 / F$ & Embryonal carcinoma & Rt BCV & Y graft & 10 & Patent & 11 & + \\
\hline & & & Lt BCV & & 8 & Occlusive & 5 & \\
\hline
\end{tabular}

$B C V$, Brachiocephalic vein; PTFE, polytetrafluoroethylene; $R t$, right; $L t$, left.

*Patients who underwent preoperative chemoradiotherapy.

\section{Patients and Methods}

From 1986 through 2001, 20 patients with malignant mediastinal tumors invading the SVC underwent surgical resection at Osaka University Hospital. Two patients did not undergo a postoperative evaluation of graft patency and thus were excluded from the final analysis. Five patients had SVC syndrome before the operation. Clinicopathologic features of the patient population are summarized in Table 1.

\section{Surgical Procedure}

Resection of the mediastinal tumors was performed through a median sternotomy in all patients. A lobectomy or pulmonary wedge resection was performed in 13 patients, and a partial pericardiectomy was performed in 7 patients. Additionally, 5 patients underwent concomitant phrenic nerve resection with diaphragm plication. Cardiopulmonary bypass was used in 3 patients with tumor invasion of the right arterial wall to allow for resection of right atrial tissue. ${ }^{5}$

\section{Right and Left BCV Reconstruction Procedures}

The surgical procedures used are shown in Figure 1. If subclavian venous pressure was greater than $40 \mathrm{~cm} \mathrm{H}_{2} \mathrm{O}$ after SVC clamping or if the SVC clamping time without circulatory support was anticipated to be greater than 45 minutes, reconstruction of the left BCV with a PTFE graft or a temporary bypass was performed, followed by reconstruction of the right $\mathrm{BCV} .^{3,6}$ Seven patients underwent reconstruction of the right $\mathrm{BCV}$ alone with a PTFE graft, whereas reconstruction was limited to the left BCV in 3 patients. Reconstruction of both the right and left BCVs was performed in 8 patients ( 6 patients received 2 separate grafts, and 2 patients received a Y graft). Anticoagulation was achieved with oral warfarin (titrated to achieve a prothrombin time/international normalized ratio of 1.8-2.0) for at least 6 months postoperatively in all patients.

\section{Results}

\section{Operative Mortality and Morbidity}

By resection of the large mediastinal veins, tumors were completely removed from 16 of 18 patients. One of the remaining 2 patients had pleural dissemination, and the other had a residual tumor in the aortic wall. No operative mortality was observed.

Operative complications occurred in 3 patients. One patient had acute mediastinitis and subsequently underwent an omentopexy procedure and was discharged 3 months after the initial operation. Two patients had intrathoracic hemorrhage, and a rethoracotomy procedure was required. Late complications were observed in 3 patients. Two patients who underwent reconstruction of the left BCV alone had 


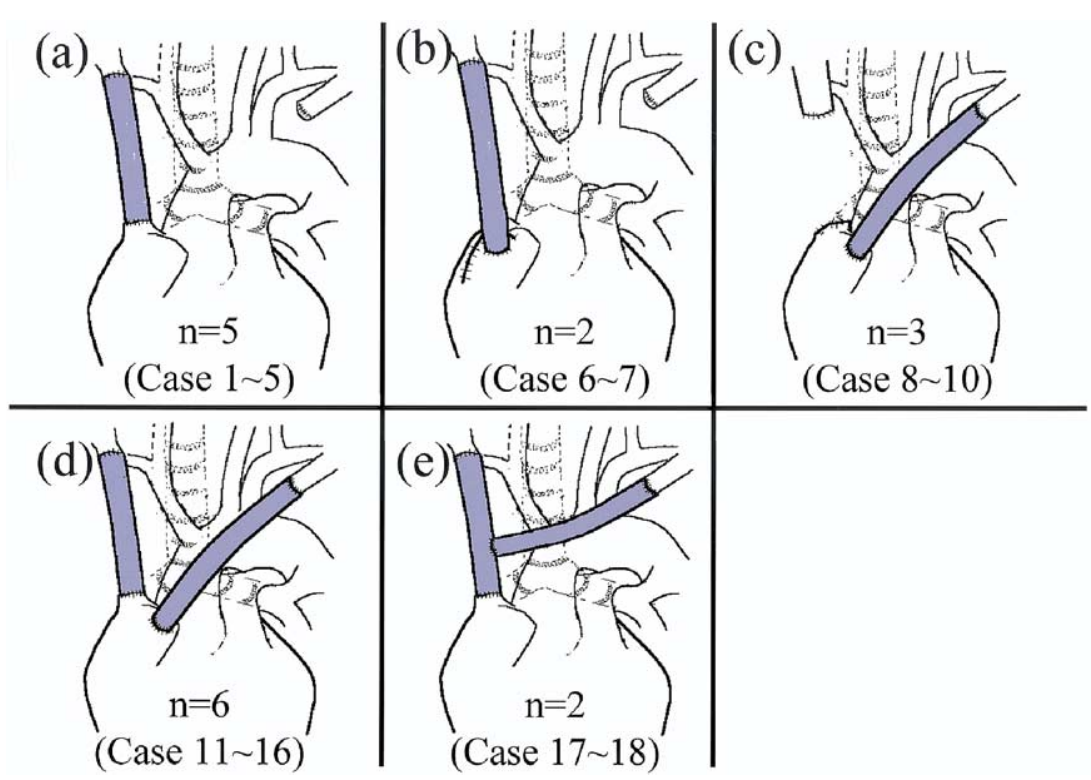

Figure 1. Types of SVC reconstruction performed in the present series. Case number corresponds with that in Table 1. a, Right BCV reconstructed with an interposed PTFE graft with proximal and distal end-to-end anastomoses between the right BCV and the SVC stump $(n=5)$. b, Graft anastomosed between the right BCV and the right atrial appendage $(n=2)$. c, Left BCV reconstructed with interposed grafts between the left BCV and the right atrial appendage $(n=3)$. d, Right and left BCVs reconstructed with 2 separate grafts $(n=6)$, in which the right BCV was reconstructed with an interposed PTFE graft with proximal and distal end-to-end anastomoses between the right BCV and the SVC stump, and the left BCV reconstruction was performed between the left BCV and the right atrial appendage. e, Right and left BCVs reconstructed with a $Y$ graft $(n=2)$.

SVC syndrome at 6 and 10 months postoperatively, respectively, and 1 patient who underwent reconstruction with a $\mathrm{Y}$ graft had persistent pleural effusion at 11 months postoperatively. These 3 patients were readmitted and treated conservatively.

\section{Graft Patency}

The types of BCV reconstruction performed and graft patency results are summarized in Table 1. All patients were evaluated postoperatively with venography or computed tomographic examinations (Figure 2) at time points ranging from 3 to 77 months. The graft remained patent in 6 of 7 patients who underwent sole reconstruction of the right $\mathrm{BCV}$, whereas 2 of 3 patients who underwent sole reconstruction of the left BCV had graft occlusion. Of the 6 patients who received 2 separate grafts, 4 patients had occlusion of one graft, whereas both grafts remained patent in the other 2 patients. In the 2 patients who received a $\mathrm{Y}$ graft, the left BCV was occluded in both, and 1 of the patients also had right $\mathrm{BCV}$ occlusion.

\section{Discussion}

Ringed PTFE grafts have been used for reconstruction of the mediastinal veins with much success; however, the optimal strategy for SVC reconstruction remains controversial. ${ }^{6-8}$ The results of the present study suggest a tendency for a sole left BCV graft to be occlusive postoperatively and to cause SVC syndrome. In contrast, the right BCV graft remained patent in 6 of 7 patients who underwent sole reconstruction of the right $\mathrm{BCV}$. In the present patients there was no significant difference in the internal diameter of the grafts between right and left BCV reconstructions (Table 1). Thus the disparity between patency rates between the right and left BCVs might have resulted from comparatively slower flow velocities in the left graft because of its longer length. Therefore sole reconstruction of the left BCV should be avoided in patients undergoing resection of the SVC and left BCV.

Bilateral reconstruction of $\mathrm{BCVs}$ has been recommended to prevent significant subclavian venous hypertension and secondary lymphatic obstruction, as well as respiratory insufficiency. ${ }^{9,10}$ However, use of a $\mathrm{Y}$ anastomosis necessitates a graft-to-graft suture that promotes thrombotic occlusion. ${ }^{3}$ Of the 2 patients who received a Y graft in the present study, 1 had left BCV graft occlusion, and the other had persistent pleural effusion caused by basal occlusion of the $\mathrm{Y}$ graft. Thus use of a Y graft for SVC reconstruction should be avoided. In contrast, although 4 of 6 patients who un- 


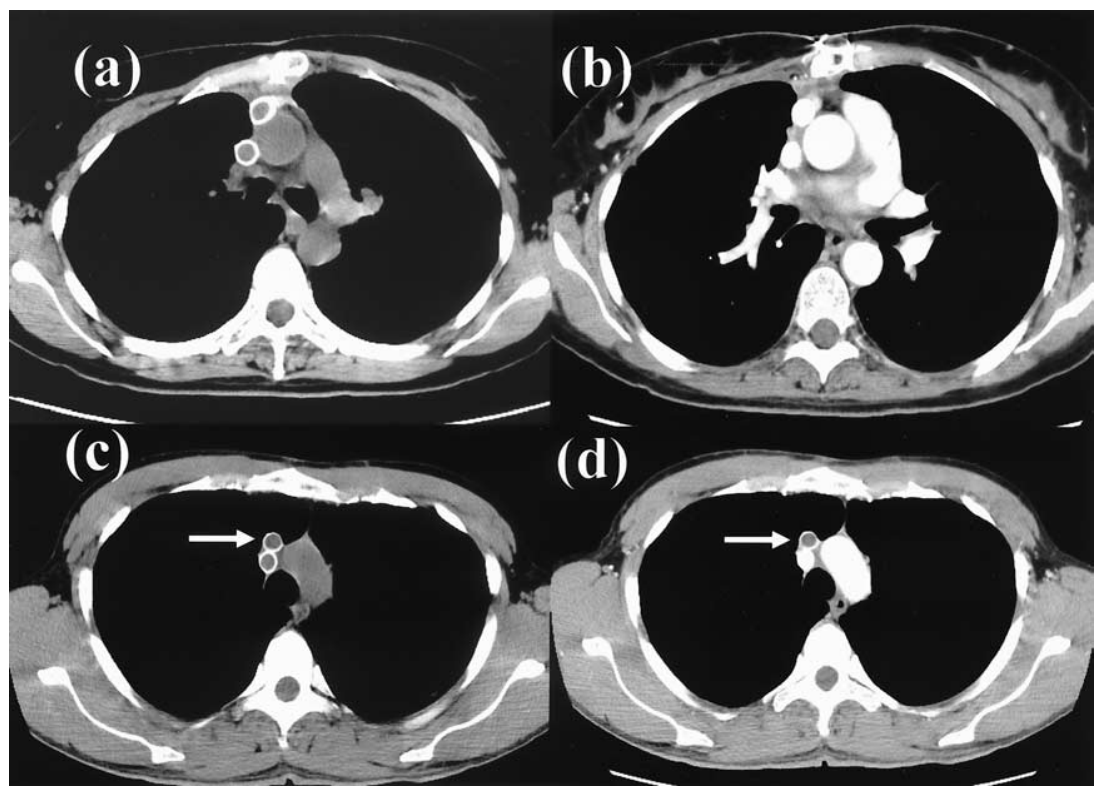

Figure 2. Representative computed tomographic images with or without contrast medium. a and b, Contrast computed tomographic images showing satisfactory patency of grafts used to replace the right and left BCVs. C and d, Contrast computed tomographic images showing patency of a graft in place of the right BCV and occlusion of a left BCV graft.

derwent bilateral separate reconstruction had occlusion of one of the grafts, occlusive symptoms were not seen in any of those patients. An optimal duration of anticoagulant therapy has not been established ${ }^{4}$; however, our results suggest that longer therapy (ie, beyond 6 months) might be necessary for patients who have undergone reconstruction of both BCVs to prevent late graft occlusion.

Five of 6 patients who underwent bilateral separate reconstruction in the present study received preoperative chemoradiotherapy (Table 1). Chemoradiotherapy results in formation of desmoplastic tissues around the tumor and makes tumor dissection extremely difficult, with increased SVC clamping time. Therefore we performed anastomoses between the left $\mathrm{BCV}$ and the right atrial appendage first to enable safe reconstruction of the right $\mathrm{BCV}$ in those patients. Another advantage of using bilateral grafts is that even if one of the bilateral grafts is occluded by thrombus formation or local tumor recurrence, the other will likely remain patent. ${ }^{7}$ Thus we advocate the use of bilateral separate reconstruction for SVC replacement.

In the present series graft occlusion was detected from 5 to 26 months postoperatively, and therefore long-term follow-up is necessary to accurately evaluate the graft patency. This can be easily accomplished by contrast-enhanced computed tomography, which is also performed to determine disease recurrence.
In conclusion, sole reconstruction of a left BCV should be avoided for SVC replacement. We advocate the use of bilateral separate reconstruction and avoidance of a $\mathrm{Y}$ graft.

\section{References}

1. Yagi K, Hirata T, Fukuse T, Yokomise H, Inui K, Ike O, et al. Surgical treatment for invasive thymoma, especially when the superior vena cava is invaded. Ann Thorac Surg. 1996;61:521-4.

2. Shimizu N, Moriyama S, Aoe M, Nakata M, Ando A, Teramoto S. The surgical treatment of invasive thymoma. Resection with vascular reconstruction. J Thorac Cardiovasc Surg. 1992;103:414-20.

3. Dartevelle P, Chapelier A, Navajas M, Levasseur P, Rojas A, Khalife J, et al. Replacement of the superior vena cava with polytetrafluoroethylene grafts combined with resection of mediastinal-pulmonary malignant tumors. Report of thirteen cases. J Thorac Cardiovasc Surg. 1987;94:361-6.

4. Dartevelle PG, Chapelier AR, Pastorino U, Corbi P, Lenot B, Cerrina $\mathrm{J}$, et al. Long-term follow-up after prosthetic replacement of the superior vena cava combined with resection of mediastinal-pulmonary malignant tumors. J Thorac Cardiovasc Surg. 1991;102:259-65.

5. Funakoshi Y, Ohta M, Maeda H, Matsuda H. Extended operation for invasive thymoma with intracaval and intracardiac extension. Eur J Cardiothorac Surg. 2003;24:331-3.

6. Nakahara K, Ohno K, Mastumura A, Hirose H, Mastuda H, Nakano S, et al. Extended operation for lung cancer invading the aortic arch and superior vena cava. J Thorac Cardiovasc Surg. 1989;97:428-33.

7. Shimizu N, Date H, Moriyama S, Ando A, Teramoto S. Reconstruction of the superior vena cava in patients with mediastinal malignancies. Eur J Cardiothorac Surg. 1991;5:575-8.

8. Lai WW, Wu MH, Chou NS, Lin MY. Surgery for malignant involvement of the superior vena cava. J Formos Med Assoc. 1992;91:991-5.

9. Nieto AF, Doty DB. Superior vena cava obstruction: clinical syndrome, etiology, and treatment. Curr Probl Cancer. 1986;10:441-84. 